\title{
Detection and first molecular characterization of bovine papular stomatitis virus in dairy calves in Argentina
}

\author{
Juan F. Micheloud ${ }^{1,2}\left(\mathbb{D} \cdot\right.$ Laura S. Aguirre $^{2} \cdot$ Gabriela V. Sandoval $^{2} \cdot$ Agustín Avellaneda-Cáceres $^{2} \cdot$ Julian Diodati $^{3} \cdot$ \\ Andrea Peralta ${ }^{4}$
}

Received: 17 March 2019 / Accepted: 4 July 2019

(C) Springer Nature B.V. 2019

\begin{abstract}
Bovine papular stomatitis virus (BPSV) is a parapoxvirus associated with papular and erosive lesions on the muzzle, lips, and oral mucosa of cattle. BPSV infection occurs worldwide; however, it has still not been unequivocally diagnosed. The present report describes an outbreak of BPSV infection affecting dairy calves in northwestern Argentina and provides the first molecular characterization of this virus in the country. The disease was detected in a dairy farm, affecting 33 calves between 2 and 20 days of age. The signs included reddish papules, ulcers, and scabby proliferative lesions on muzzle, lips, and oral mucosa. The affected calves resisted to being fed due to severe local pain. Two necropsies were performed; papulas and ulcers were observed in ruminal and omasal mucosa. Histologically, the affected areas of the skin showed acanthosis, spongiosis, and parakeratotic hyperkeratosis with adjacent focally extensive ulcers and multifocal inflammatory infiltrate in the epidermis. Eosinophilic inclusion bodies were detected in the cytoplasm of epithelial cells. DNA extracted from scab samples was analyzed by PCR using pan-parapoxvirus primers for the B2L gene. The sequence analysis revealed $99 \%, 85 \%$, and $84 \%$ similarity with BPSV, Pseudocowpox virus, and Orf virus, respectively. A phylogenetic tree constructed using the B2L sequence showed that the virus clustered with BPSV isolates. Although clinical cases compatible with BSPV infection have been frequently described in Argentina, the present report is the first to identify the agent associated with cattle disease in the country.
\end{abstract}

Keywords Bovine papular stomatitis virus $\cdot$ Cattle $\cdot$ Cutaneous disease $\cdot$ Parapoxvirus

Bovine papular stomatitis (BPS) is a common viral disease of calves characterized by the formation of papules or nodules that progress to vesicles and then crusts or scabs on the lips, gingiva, and tongue (Mark et al. 1991). The disease is caused by bovine papular stomatitis virus (BPSV) (Mayr and Büttner 1990). BPSV is a Parapoxvirus (PPV) belonging to the family

Juan F. Micheloud

micheloud.juan@inta.gob.ar

1 Grupo de Trabajo de Patología, Epidemiología e Investigación Diagnóstica. Área de Sanidad Animal-IIACS, Instituto Nacional de Tecnología Agropecuaria (INTA), Cerrillos, Salta, Argentina

2 Cátedra Práctica Hospitalaria de Grandes Animales, Universidad Católica de Salta, Salta, Argentina

3 Laboratorio Integral de Microscopía, CICVyA, INTA, Hurlingham, Argentina

4 Instituto de Agrobiotecnología y Biología Molecular (IABIMO), INTA, Consejo Nacional de investigaciones Científicas y Tecnológicas (CONICET), Hurlingham, Argentina
Poxviridae, subfamily Chordopoxvirinae (Büttner and Rziha 2002). PPVs include four species: BPSV, Pseudocowpoxvirus (PCPV), parapoxvirus of red deer in New Zealand (PVNZ), and Orf virus (ORFV), which is the prototype of PPV. PPVs are epitheliotropic viruses that cause nonsystemic, proliferative skin disease in domestic and wild ruminants (Mark et al. 1991; Delhon et al. 2004). BPSV infection in young cattle is sometimes manifested with distinctive "horseshoe-shaped" popular lesions on the hard palate and oral mucosa, which can occur with or without concurrent inflammation of the gingiva (Dal Pozzo et al. 2011). BPS is widespread around the world and can occasionally be transmitted to humans (de Sant'Ana et al. 2012). In humans, BPSV infection is associated with nodules and pustules on the hands and sometimes on the face (Bowman et al. 1981). In South America, cases clinically compatible with BPSV infection have been frequently reported by veterinarians, and laboratory-confirmed cases have been reported in Uruguay and Brazil (Lemos and RietCorrea 2007; de Sant'Ana et al. 2012). In Argentina, a BPS case was described by Rodriguez et al. (1988), but the agent 
has still not been unequivocally diagnosed. In this study, we report an outbreak of BPSV infection in cattle and provide the first molecular characterization of this virus for country.

In September 2018, 28 of 33 dairy calves housed on a farm in Salta province presented mildly erosive papules, coalescent scabby erosions, and ulcers mainly on the muzzle and oral cavity (Fig. 1). The affected farm held cattle for dairy production. Mean temperature at the time of the outbreak was approximately $22{ }^{\circ} \mathrm{C}$, and the weather was dry and clean. Disease morbidity was $85 \%$ (28/33), and the affected calves were between 2 and 20 days old. Mortality was high $(67 \% ; 23 /$ 33). They resisted to being milked due to severe local pain. In addition, some animals had diarrhea and dehydration. Two recently dead calves were available for post-mortem examination. Necropsy revealed proliferative lesions and coalescent scabby erosions in oral mucosa. Additionally, in one calf, the ruminal and omasal mucosa were severe affected. Other animals recovered without treatment 15-18 days after the first appearance of clinical signs. On the farm, there were 250 adult cows in production; all of them were inspected for lesions compatible with BPS during milking. No cows presented lesions, suggesting the absence of clinical manifestation.

Clinical samples of the noses and lips of affected calves were collected. For histopathology studies, samples of fresh tissue were fixed in $10 \%$ buffered formalin, processed for routine histopathological examination, and stained with hematoxylin and eosin. Histologically, multifocal areas of moderate acanthosis, spongiosis, and parakeratotic hyperkeratosis with adjacent focally extensive ulcers were identified in the epidermis (Fig. 1c). In the cytoplasm of epithelial cells of areas with degenerative changes numerous $2-6-\mu \mathrm{m}$ circular, eosinophilic inclusion bodies were detected (insert in Fig. 1c). In more
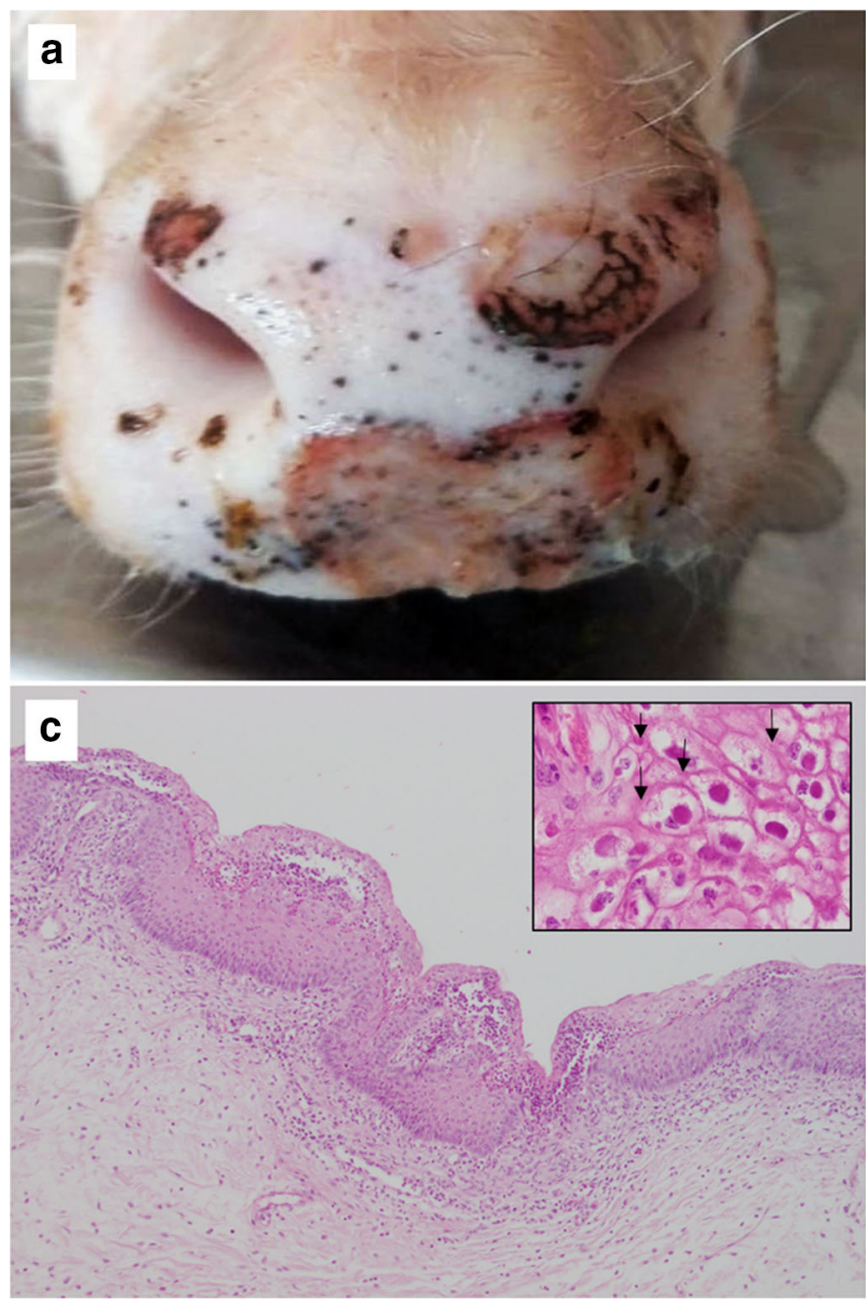

Fig. 1 Calves infected with BPSV-Salta. a Multiple ulcerative and erosive lesions in the nose and lips. b Typical lesions of disease on the palate. c Microphotography of lips. Pustular and necrotizing dermatitis with severe diffuse proliferative acanthosis and moderate
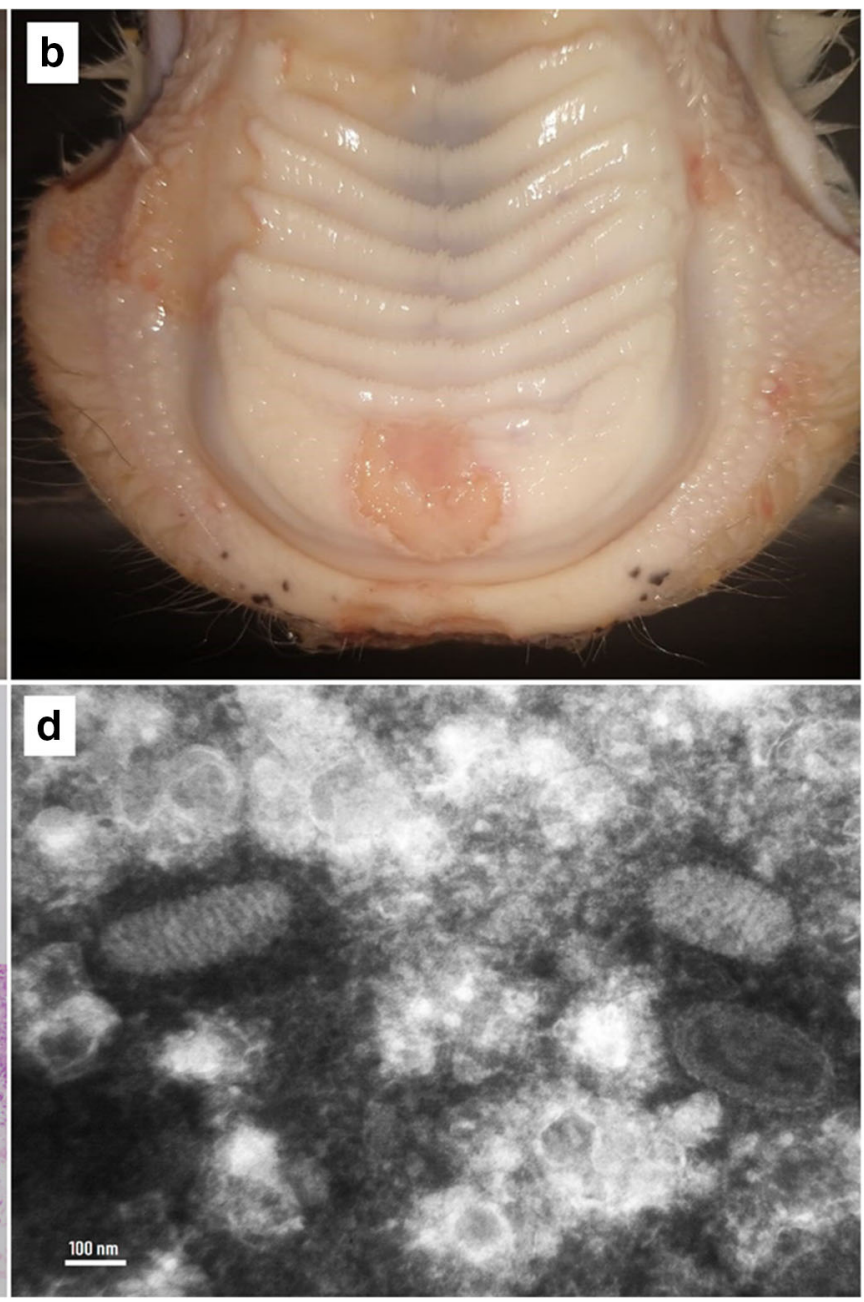

lymphoplasmacytic infiltration. The insert shown ballooning degeneration of superficial keratinocytes and typical viral inclusion bodies (arrows) (H\&E 20×). d Electron micrograph of a BPSV particle showing the typical helix-shaped morphology $($ bar $=100 \mathrm{~nm})$ 


\section{a}

BV-ARO2 (BPSV) BPSV C5 (BPSV) V660 (BPSV) Iwate (BPSV) Ishikawa (BPSV) SV819/10 (BPSV) SV716-12 (BPSV) BPSV Salta NEL (UREV) IA82 (ORFV) SPi13 (ORFV) VR634 (PCPV) Tillquist (PCPV)

BV-ARO2 (BPSV) BPSV C5 (BPSV) V660 (BPSV) Iwate (BPSV) Ishikawa (BPSV) SV819/10 (BPSV) SV716-12 (BPSV) BPSV Salta NZ2 (ORFV) IA82 (ORFV) SPi13 (ORFV) VR634 (PCPV) Tillquist (PCPV)

BV-ARO2 (BPSV) BPSV C5 (BPSV) V660 (BPSV) Iwate (BPSV) Ishikawa (BPSV) SV819/10 (BPSV) SV716-12 (BPSV) BPSV Salta NZ2 (ORFV) IA82 (ORFV)

SPi13 (ORFV) VR634 (PCPV) Tillquist (PCPV)

BV-ARO2 (BPSV) BPSV O5 (BPSV) V660 (BPSV)

Iwate (BPSV)

Ishikawa (BPSV) SV819/10 (BPSV) SV716-12 (BPSV)

BPSV Salta NZ2 (ORFV) IA82 (ORFV)

SPi13 (ORFV) VR634 (PCPV) Tillquist (PCPV)

BV-AR02 (BPSV) BPSV C5 (BPSV) V660 (BPSV) Iwate (BPSV) Ishikawa (BPSV) SV819/10 (BPSV) SV716-12 (BPSV) BPSV Salta NZ2 (ORFV)

Fig. 2 Molecular characterization of BPSV-Salta based on internal region of B2L gene. a Alignment of nucleotide sequences from internal region of B2L gene among BPSV-Salta and several parapoxvirus: BPSV, ORFV, and PCPV. b Phylogenetic analysis of Argentine BPSV outbreak based on $\mathrm{B} 2 \mathrm{~L}$ gene. The phylogenetic relationships were constructed by the

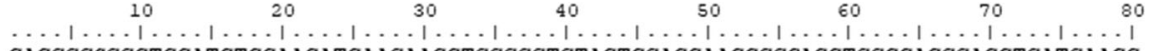
CACGGGCGGCTCCATCTCCAACATCAAGAACCTGGGGTGTACTCGACCAACCGGCACCTGGCCACGGACCTGATGAACC









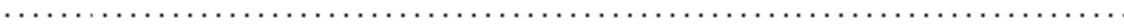

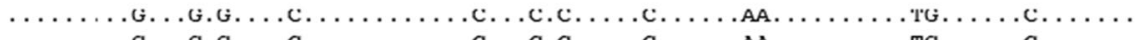
$\ldots \ldots$.

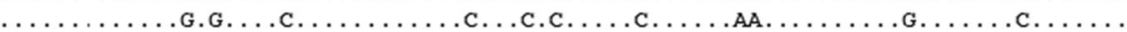

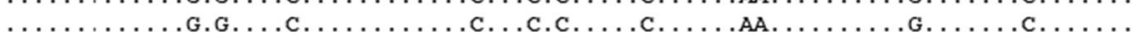

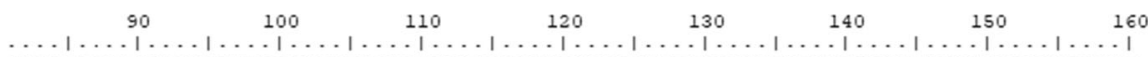
GGTACAACACCTTCTACTCCATGATCGTGGAGCCCAAGGTCCCTTCTCGCGGCTGTGCTGCGCCATGATCACGCCCACG





.



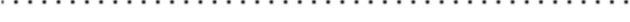

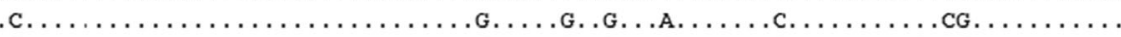

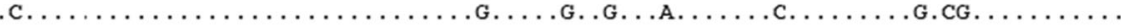

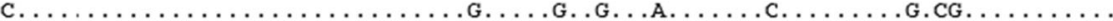

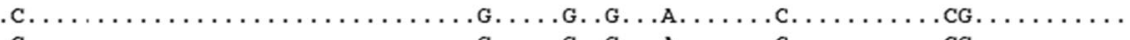

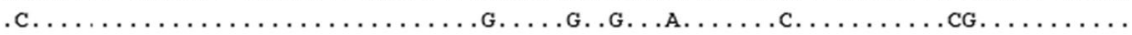

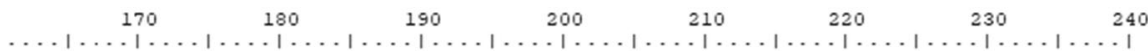
GCAACGGACTTCCACCTGGACCACGCGGGCGGCGGCGTGTTCTTCTCGGACGCGCCCGAGAAGTTCCTGGGCTTCTACCG

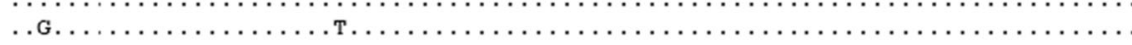

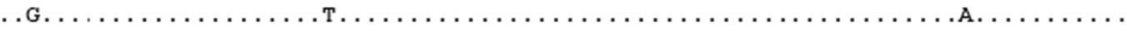

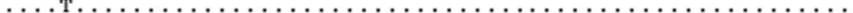

.

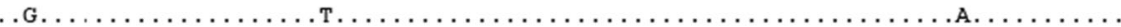

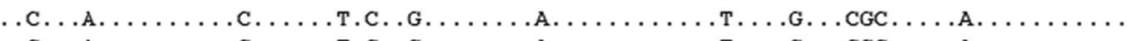

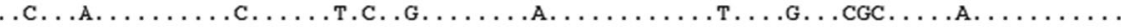

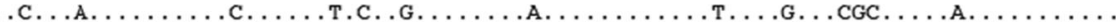

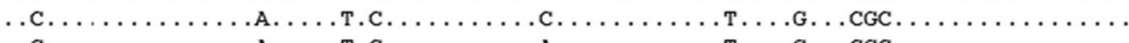

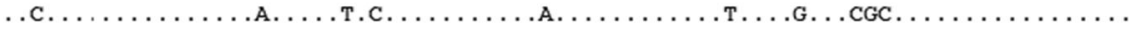

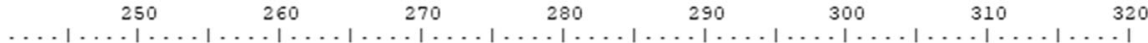
CACGCTGGACGAGGACCTGGTGCTGCACCGCATCGACTCCGCGAAGAACAGCATCGACCTGTCGCTGCTGTCCCTGGTGC

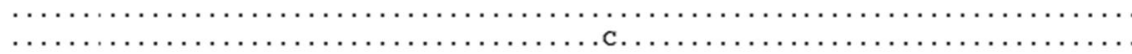





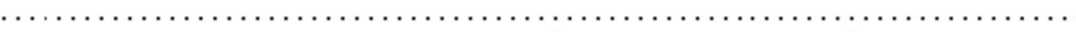

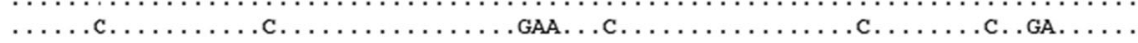

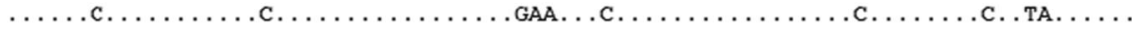

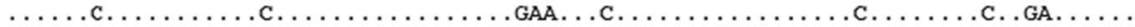

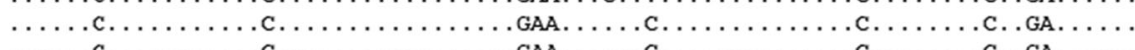

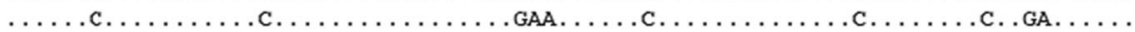

$350 \quad 360 \quad 370 \quad 380-390 \quad 400$ CCGTGATCCGGCACGCCGACCGCGTGGAGTACTGGCCGCGGATCATGGACGCGTTGCTGCGCGCGGCCATCGACCGCAGC

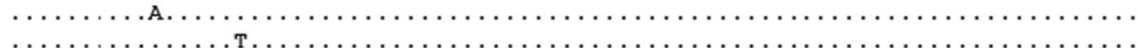

…

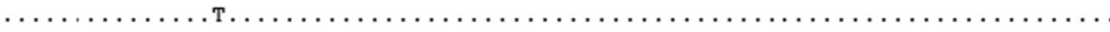

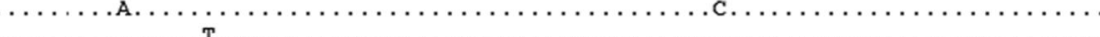

.

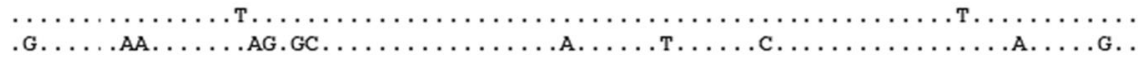

neighbor-joining algorithm using MEGA 6.0 software. All positions with less than $50 \%$ site coverage were removed. Numbers at nodes represent percentage of 1000 bootstrap replicates. Black circle pointed to BPSVSalta 


IA82 (ORFV)
SPi13 (ORFV)
VR634 (PCPV)
Tillquist (PCPV)

BV-AR02 (BPSV)
BPSV c5 (BPSV)
V660 (BPSV)
Iwate (BPSV)
Ishikawa (BPSV)
SV819/10 (BPSV)
SV716-12 (BPSV)
BPSV Salta
NZ2 (ORFV)
IA82 (ORFV)
SPi13 (ORFV)
VR634 (PCPV)
Tillquist (PCPV)

BV-AR02 (BPSV)
BPSV C5 (BPSV)
V660 (BPSV)
Iwate (BPSV)
Ishikawa (BPSV)
SV819/10 (BPSV)
SV716-12 (BPSV)
BPSV Salta
NZ2 (ORFV)
IA82 (ORFV)
SPi13 (ORFV)
VR634 (PCPV)
Tillquist (PCPV)

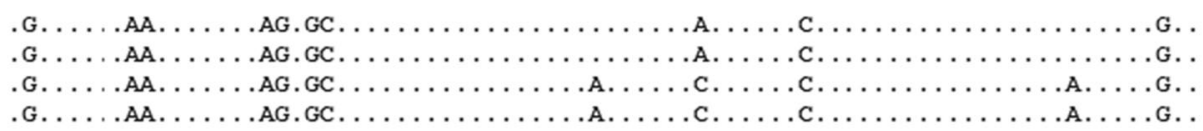

Fig. 2 continued.

affected areas, tissues of the superficial dermis presented moderate inflammatory infiltrate of lymphocytes, neutrophils, and macrophages. The adjacent dermis showed the same type of perivascular infiltrate.

Parapoxvirus infections are usually considered diseases that should be differentiated from important vesicular diseases in ruminants. Therefore, we extracted DNA (QIAamp DNA mini kit, QIAGEN) and RNA (Trizol Reagent, Invitrogen) from scab samples to determine the etiological agent of the lesion. The presence of bovine viral diarrhoea virus (BVDV) was discounted by RT-PCR based on 5'UTR BVDV genome sequence (Pellerin et al. 1994), foot-and-mouth disease virus (FMDV) was rejected by RT-PCR based on 3D gene sequence, and vesicular stomatitis virus (VSV) was discounted by RT-PCR based on an internal region of VSV glycoprotein G gene sequence. Possible Orthopoxvirus infection was discounted by pan-pox low-GC PCR assay (Li et al. 2010), whereas pan-pox high-GC PCR indicated a member of PPVs as responsible for the animal injury (data not shown).

Tissue samples were homogenized mechanically in phosphate-buffered saline (PBS) using a pellet pestle device. Homogenates were then centrifuged at $3000 \times g$ for $5 \mathrm{~min}$, and the supernatant was loaded on 200 mesh copper grid and stained with $2 \%$ phosphotungstic acid for transmission electron microscopic examination. This study revealed typical
PPV particles: oval $(260 \mathrm{~nm} \times 160 \mathrm{~nm})$, enveloped, and with a helical structure (Fig. 1d).

Once PPV was confirmed as the etiological agent, a PCR using pan-parapoxvirus primers for the B2L gene (Inoshima et al. 2000) was performed. The amplified 590-bp product was directly sequenced in both directions using the amplification primers. Genomic information was derived from overlapping sequences covered by the forward and reverse primers. The sequence obtained was aligned with BPSV, ORFV, and PCPV parapoxvirus sequences available in the GenBank (Fig. 2a). In addition to the BPS case, ORFV sequences obtained from previous cases occurred in the same Argentine province were also included. The identity matrix revealed a nucleotide identity of $99 \%, 85 \%$, and $84 \%$ with BPSV, PCPV, and ORFV, respectively. The sequence was called "Salta" and was submitted to the GenBank databases under accession number MK285566.

Given that our neighboring country Brazil has several reports on outbreaks and characterization of BPSV strains, we decided to determine if the BPSV-Salta was related to Brazilian strains. Then, a phylogenetic analysis was performed using the neighbor-joining method with 1000 bootstrap replicates, using MEGA software version 6 (Tamura et al. 2013), and the Tamura-3 parameters (Tamura and Nei 1993) were selected as the fitted evolution model. As expected, the NJ tree showed that BPSV-Salta clustered with other 


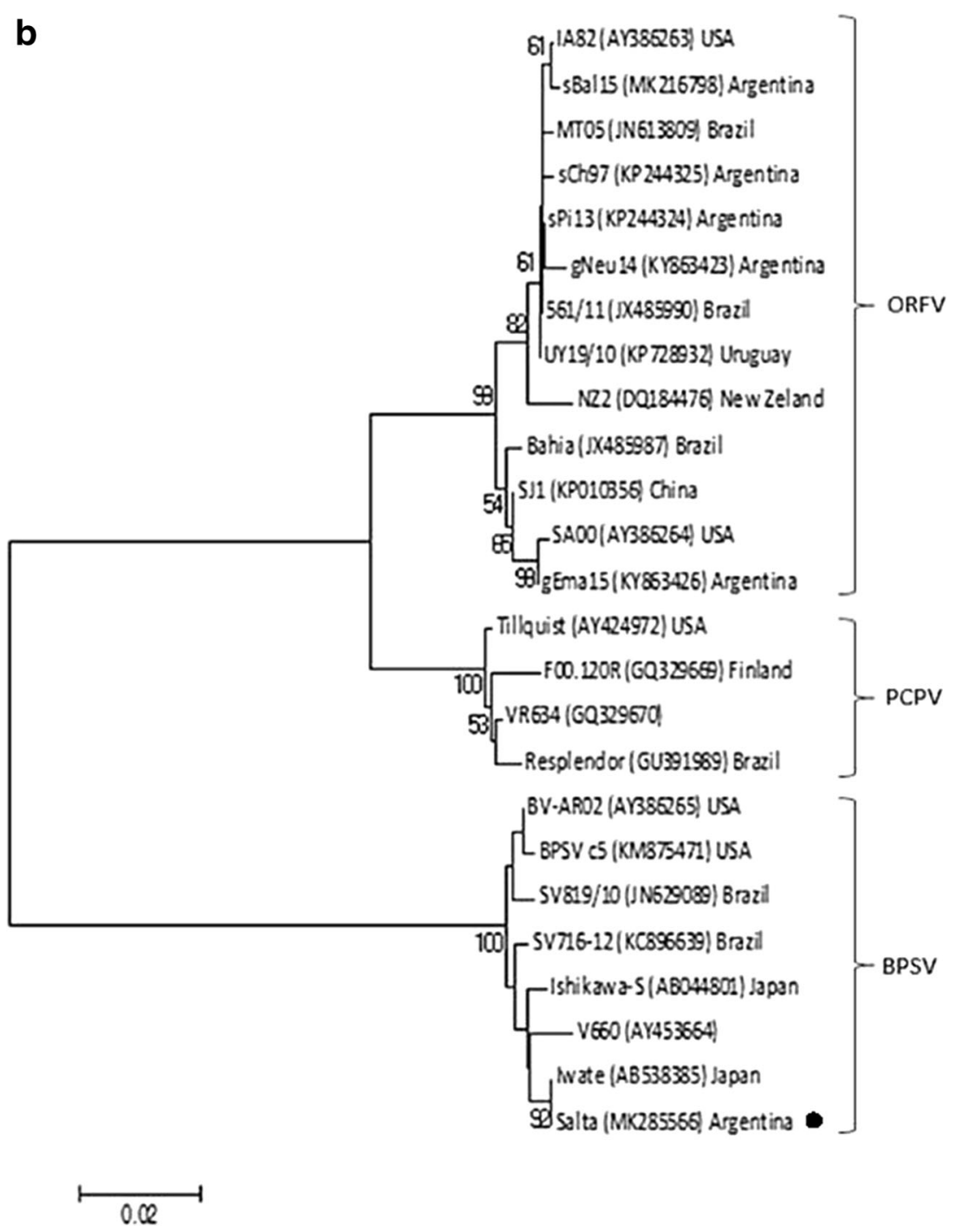

Fig. 2 continued.

BPSV isolates, with high bootstrap value, and separated from PCPV and ORFV strains (Fig. 2b). BPSV-Salta showed 100\% identity with Iwate strain (from Japan) and 98.9\% identity with Brazilian and North American BPSV strain (see Fig. $2 b)$. Given that there is no import record of cattle from Japan to Argentina, we hypothesize that this observation is due to a low phylogenetic signal caused by the high degree of identity in this internal region of the $\mathrm{B} 2 \mathrm{~L}$ gene.

This sequence was able to distinguish viral species within the PPV genus. However, within each viral species node, many of the branches have nodes with low or intermediate bootstrap values. This phenomenon was also reported in ORFV and BPSV phylogenetic studies (Schmidt et al. 2013; Billinis et al. 2012; Li et al. 2013; Oem et al. 2013; Yaegashi et al. 2013; Peralta et al. 2018). This effect could be caused by the high level of identity observed within these gene sequences, which results in a low phylogenetic signal among isolates from different countries and continents.

The clinical and pathological findings are similar to those reported for other BPS outbreaks (de Sant'Ana et al. 2012; Dal Pozzo et al. 2011; Jeckel et al. 2011). Findings include papules, often mildly erosive mainly on the muzzle, lips, hard palate, and oral mucosa of calves and, occasionally, on the tongue, esophagus, and forestomach (Brown et al. 2007). In both necropsied calves, the esophagus was unaffected; however, ruminal and omasal mucosa presented necrotic and proliferative lesion. Parapoxvirus infection generally occurs through scarified or damaged skin, followed by virus replication in keratinocytes (Büttner and Rziha 2002). Histologically, this infection produced acanthosis, spongiosis, and 
parakeratotic or orthokeratotic hyperkeratosis with adjacent focally extensive ulcers in the epidermis. In early lesions, eosinophilic inclusion bodies were noted in the cytoplasm of epithelial cells of areas with acanthosis or necrosis (Brown et al. 2007).

In this report, the control of the outbreak was carried out through biosecurity and hygiene measures. Infected calves were separated by a physical division on one side and healthy newborns on the other. Using gloves and other biosecurity attire was indicated for employees in charge of caring for the calves. BPS is considered a neglected zoonosis and several case reports in humans were previously reported (de Sant'Ana et al. 2012; Bowman et al. 1981).

Parapoxvirus infections are usually considered important diseases that should be differentiated from other vesicular diseases in ruminants, such as infection by foot-and-mouth virus. However, BPS does not have to be reported to the World Organization for Animal Health (OIE). On the other hand, the presence and wide distribution of parapoxvirus infection in cattle and humans have been frequently reported (Büttner and Rziha 2002; Dal Pozzo et al. 2011; Inoshima et al. 2009; Jeckel et al. 2011; de Sant'Ana et al. 2012; Oem et al. 2013; Lederman et al. 2014). In addition, severe atypical cases of parapoxvirus infection have been recently reported (Inoshima et al. 2009; Leonard et al. 2009; Jeckel et al. 2011). Therefore, the importance of parapoxvirus infection in cattle should be re-evaluated. Further studies are needed to determine the prevalence of infection and to identify risk factors for both animals and humans.

Acknowledgments We thank Dr. Chimeno, Dr. Amalfi, and Dr. Konig (INTA) for kindly providing us with the primers and positive controls for the RT-PCR assays used in this work.

Funding information Financial support was provided by grants PNBIO1131032 and PNSA1115055 from INTA (Instituto Nacional de Tecnología Agropecuaria) and Consejo de Investigación de la Universidad Católica de Salta.

\section{Compliance with ethical standards}

Conflict of interest The authors declare that they have no conflicts of interest.

\section{References}

Billinis, C., Mavrogianni, V.S., Spyrou, V., Fthenakis, G.C., 2012. Phylogenetic analysis of strains of Orf virus isolated from two outbreaks of the disease in sheep in Greece. Virology Journal, 9, 24.

Bowman, K.F., Barbery, R.T., Swango, L.J., Schnurrenberger, P.R., 1981. Cutaneous form of bovine papular stomatitis in man. Journal of the American Medical Association, 246, 2813-2818.

Brown, C.C., Baker, D.C., Barker, I.K., 2007. Alimentary system. In: Jubb, Kennedy, and Palmer's pathology of domestic animals, ed. Maxie MG, 5th ed., pp. 1-296. Saunders Elsevier, New York, NY.
Büttner, M., Rziha, H.J., 2002. Parapoxviruses: from the lesion to the viral genome. Journal of veterinary medicine / Series B, Infectious diseases, immunobiology, food hygiene, public health, 49,7-16.

Dal Pozzo, F., Martinelle, L., Gallina, L., Mast, J., Sarradin, P., Thiry, E., Scagliarini, A., Büttner, M., Saegerman, C., 2011. Original findings associated with two cases of bovine popular stomatitis. Journal of Clinical Microbiology, 49, 4397-4400.

de Sant'Ana, F.J., Rabelo, R.E., Vulcani, V.A., Cargnelutti J.F., Flores E.F., 2012. Bovine papular stomatitis affecting dairy cows and milkers in midwestern Brazil. Journal of Veterinary Diagnostic Investigation, 24: 442-445.

Delhon, G., Tulman, E.R., Afonso, C.L., Lu, Z., de la Concha-Bermejillo, A., Lehmkuhl, H.D., Piccone, M.E., Kutish, G.F., Rock, D.L., 2004. Genomes of the parapoxviruses ORF virus and bovine papular stomatitis virus. Journal of Virology, 78, 168-177.

Inoshima, Y., Morroka, A., Sentsui, H., 2000. Detection and diagnosis of parapoxvirus by the polymerase chain reaction. Journal of Virological Methods, 84, 201-208.

Inoshima, Y., Nakane, T., Sentsui, H., 2009. Severe dermatitis on cattle teats caused by bovine papular stomatitis virus. Veterinary Record, $164,311-312$.

Jeckel, S., Bidewell, C., Everest, D., McInnes, C., Wood, A., Dare, J., Schock, A., 2011. Severe oesophagitis in an adult bull caused by bovine papular stomatitis virus. Veterinary Record, 169, 317.

Lederman, E., Khan, S.U., Luby, S., Zhao, H., Braden, Z., Gao, J., Karem, K., Damon, I., Reynolds, M., Li, Y., 2014. Zoonotic parapoxviruses detected in symptomatic cattle in Bangladesh. BMC Research Notes, 7, 816.

Lemos, R.A.A. and Riet-Correa, F., 2007. Infecções víricas da pele do úbere em bovinos [Viral infections of the skin of the udder in cattle]. In: Doenças de ruminantes e equídeos [Diseases of ruminants and horses], ed. Riet-Correa F, Schild AL, Lemos RAA, Borges JRJ, 3rd ed., pp. 147-152. Pallotti, Santa Maria, Brazil. In Portuguese.

Leonard, D., Otter, A., Everest, D., Wood, A., McInnes, C., Schock, A., 2009. Unusual bovine papular stomatitis virus infection in a British dairy cow. Veterinary Record, 164, 65.

Li, Y., Meyer, H., Zhao, H., Damon, I.K., 2010. GC content-based PanPox universal PCR assays for poxvirus detection. Journal of Clinical Microbiology, 48, 268-272.

Li, H., Zhu, X., Zheng, Y., Wang, S., Liu, Z., Dou, Y., Li, H., Cai, X., Luo, X., 2013. Phylogenetic analysis of two Chinese Orf virus isolates based on sequences of B2L and VIR genes. Archives of Virology, $158,1477-85$.

Mark, R., Buller, L., Palumbo, G.J., 1991. Poxvirus pathogenesis. Microbiology Reviews, 55, 80-122.

Mayr, A., and M. Büttner. (1990). Bovine papular stomatitis virus, p. 23 28. In Z. Dinter and B. Morein (ed.), Virus infections of ruminants. Elsevier, Amsterdam, The Netherlands.

Oem, J.K., Lee, E.Y., Lee, K.K., Keem, S.H., Lee, M.H., Hyun, B.H., 2013. Bovine Papular Stomatitis Virus (BPSV) infections in Korean native cattle. Veterinary Medicine and Science, 75, 675-678.

Pellerin, C., Van Den Hurk, J., Lecomte, J., Tussen, P., 1994. Identification of a new group of bovine viral diarrhea virus strains associated with severe outbreaks and high mortalities. Virology, 203, 260-268.

Peralta, A., Robles, C., Micheloud, J. F, Rossanigo, C., Martinez, A., Carosio, A., Konig, G., 2018. Phylogenetic analysis of ORF viruses from five Contagious Ecthyma outbreaks in Argentinian goats. Frontiers in Veterinary Science, 5, 134.

Rodriguez A.R., Gollan, A., Pinotti, M., Baroni, A.C. de, Garcia, M. 1988. Bovine papular stomatitis. First isolation of the causal agent in Argentina and experimental reproduction of the disease. Veterinaria Argentina, 18, 20-21.

Schmidt, C., Cargnelutti, J.F., Brum, M.C.S., Traesel, C.K., Weiblen, R., Flores, E.F., 2013. Partial sequence analysis of B2L gene of 
Brazilian orf viruses from sheep and goats. Veterinary Microbiology, $162,245-253$.

Tamura, K. and Nei, M., 1993. Estimation of the number of nucleotide substitutions in the control region of mitochondrial DNA in humans and chimpanzees. Molecular Biology and Evolution, 10, 512-526.

Tamura, K., Stecher, G., Peterson, D., Filipski, A., Kumar, S., 2013. MEGA6: Molecular Evolutionary Genetics Analysis version 6.0. Molecular Biology and Evolution, 30, 2725-2729.
Yaegashi, G., Sasaki, I., Chiba, S., Murakami, K., 2013. Molecular analysis of Parapoxvirus detected in eight calves in Japan. Journal of Veterinary Medical Science, 75, 1399-1403.

Publisher's note Springer Nature remains neutral with regard to jurisdictional claims in published maps and institutional affiliations. 